\title{
Emergency management of ludwig's angina: a case report
}

\author{
Nur H. Alimin*, Endang Syamsuddin
}

\section{Abstract}

Objective: Ludwig's angina is a severe diffuse cellulitis in mandibular region that is commonly caused by odontogenic infection. Due to its acuteness at onset and rapid spread, involving the submandibular, sublingual region bilaterally and submental region, this condition is considered as an emergency because it can cause airway obstruction. Methods: A 40 years old male patient came to Dr. Hasan Sadikin Hospital emergency room with pain and swelling of lower jaw, drooling, difficulty in breathing and opening his mouth. He was diagnosed with sepsis and Ludwig's angina. Tracheostomy was performed to secure the airway, which was followed by teeth extraction, incision and drainage to eliminate the source of infection. A combination of intravenous antimicrobial medicines was administered.

Results: An advanced case of Ludwig's angina and its management was reported. The patient showed good response to the treatment and his condition improved.

Conclusion: Ludwig's angina is a rare emergency condition that is potentially life-threatening. Patient showed significant recovery due to immediate and rapid management in securing airway patency and infection source control to prevent the spread of infection and further complications.

Keywords: Infection source control, Ludwig's angina, Tracheostomy

Cite this Article: Alimin NH, Syamsuddin E. 2017. Emergency management of ludwig's angina: a case report. Journal of Dentomaxillofacial Science 2(3): 201-204. D0l: 10.15562/jdmfs.v2i3.517

Department of Oral and Maxillofacial Surgery, Faculty of Dentistry, Universitas Padjadjaran, Bandung, Indonesia

*Corresponding to: Nur H. Alimin, Department of Oral and Maxillofacial Surgery, Faculty of Dentistry, Universitas Padjadjaran, Bandung, Indonesia

fkguh06punya@gmail.com

Received: 13 July 2017

Revised: 30 0ctober 2017

Accepted: 1 November 2017

Available online: 1 December 2017

\section{Introduction}

Ludwig's angina was first discovered in 1836 by Friedrich Wilhelm von Ludwig, a surgeon from Germany; he defined the condition as a great diffuse cellulitis with an acute onset and rapid spread, involving bilateral spaces of submandibular, sublingual and submental regions. ${ }^{1,2}$ Based on research conducted in 2010-2011 with 30 patients in Pakistan, Ansari et al. ${ }^{3}$ showed that men are more frequently affected with the condition than women (3:1-4:1); the condition is more prevalent among population groups belonging to lower socioeconomic class and usually occurs in the third decade of life. Diabetes mellitus was reported as a systemic illness that often underlines this condition. Other studies showed the mortality rate of Ludwig's angina is $8-10 \%$ and that it occurs more often by asphyxia than by sepsis. ${ }^{3}$ Ludwig's angina and deep neck abscesses are an entity that could be potentially fatal due to its tendency to lead to edema, distortion and airway obstruction. ${ }^{4}$

Majority of infections spread to submandibular space caused by odontogenic infection of the lower-second or third molar with its apex located below the mylohyoid muscle, which then rapidly spreads to the sublingual space, usually on a bilateral basis, although early symptoms are usually unilateral. Clinically, swellings occur at submandible and submentale regions with hard consistency and pain. Furthermore, it could cause stiffness of the neck, trismus, odynophagia (pain on swallowing) and drooling. ${ }^{5-8}$ The development from the onset to respiratory obstruction might happen within 12-24 hours. ${ }^{9}$ Ludwig's angina is a mixed polymicrobial infection (aerobic and anaerobic), which normally colonizes in the oropharynx. The use of antibiotics have reduced mortality from $54 \%$ to $0-8.5 \%$.

The reasons for high mortality rate associated with Ludwig's angina include late diagnosis and inadequate management of airway obstruction. ${ }^{4,6}$ Therefore, Ludwig's angina is an emergency condition that is rare and potentially life-threatening if it is not recognized on time and treated aggressively.

\section{Case Report}

A male patient (40 years old) came to the emergency room (ER) of Dr. Hasan Sadikin General Hospital; he was referred from the Regional General Hospital; symptoms included limitations in opening the mouth, pain and swelling of the left-lower jaw preceded by toothache four days earlier. Anamnesis found that the swelling extended to chin and rightlower jaw two days earlier. The patient complained that it was hard for him to breath and had experienced difficulty in swallowing. The patient denied any underlying systemic abnormalities that may have led to his present condition.

Physical examination found the patient was fully alert, suffering mild dehydration with respiratory 
disruptions. Visual Analog Scale (VAS) was 6/10, drooling and sniffing position. Measurements of initial vital signs found a febrile temperature at $38.6^{\circ} \mathrm{C}$, tachycardia with $110 \mathrm{x} / \mathrm{min}$, tachypnea $26 \mathrm{x} / \mathrm{min}$, blood pressure 120/80 $\mathrm{mmHg}$, and oxygen saturation (SpO2 94\%). Extra-orally, facial asymmetry with diffuse swelling on the bilateral submandible and submental regions expanded to the left buccal and neck region; on palpation it was found that the swelling was warm, tender, and no fluctuation. Intra-orally, there was radices of teeth $28,46,47$ and pulp gangrene of tooth 37 , trismus with mouth opening limited to $2 \mathrm{~cm}$ and swelling at the floor of mouth and tongue appeared lifted figure 1. Laboratory findings showed leukocytosis (16,600 / mm3), hyperglycemic state $(191 \mathrm{mg} / \mathrm{dL})$, and hyponatremia $(131 \mathrm{mEq} / \mathrm{L})$. Neck soft tissue radiographs showed soft tissue mass in the left submandible that pushed the air column to the right figure 2. Chest $\mathrm{x}$-ray impression was within normal limits. The patient was also consulted at the Ear, Neck and Throat (ENT) Department and Internal Medicine Department as a holistic treatment plan.

The patient was diagnosed with Ludwig's angina accompanied with sepsis. The patient was planned to undergo a tracheostomy and source control under local anesthesia. Pus aspiration was done to check bacteria culture and antibiotic sensitivity.

After the tracheostomy was done, infection source control was performed. Airway and vital signs were observed closely during the treatment. Incision and drainage were done "through and through" in the bilateral submandible and submentale regions (pus drainage $\pm 10 \mathrm{cc}$ ) and sublingual region (pus drainage $\pm 5 \mathrm{cc}$ ) figure 3 . Procedure was further continued with extraction of teeth (37, 46, 47 and 28). Patient was hospitalized for 6 days. Tracheostomy canule was maintained until the 6th day. Patient showed great improvement and started as an outpatient on the 7 th day figure 4 . All the invasive treatments were performed while the patient was in the ER figure 5.
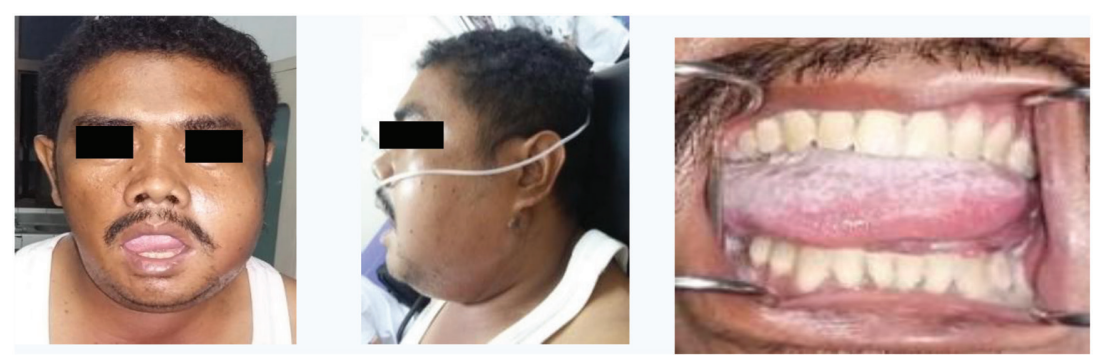

Figure 1 Preoperative, A. Extra oral, swelling on the bilateral submandible, submental, and sublingual regions with sniffing position; B. Intraoral, lifted tongue and trismus

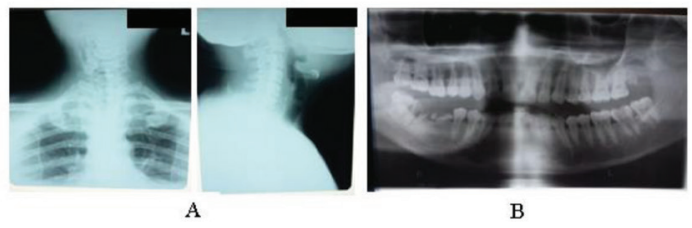

Figure 2 A. Neck soft tissue radiographs. B. Panoramic radiographs

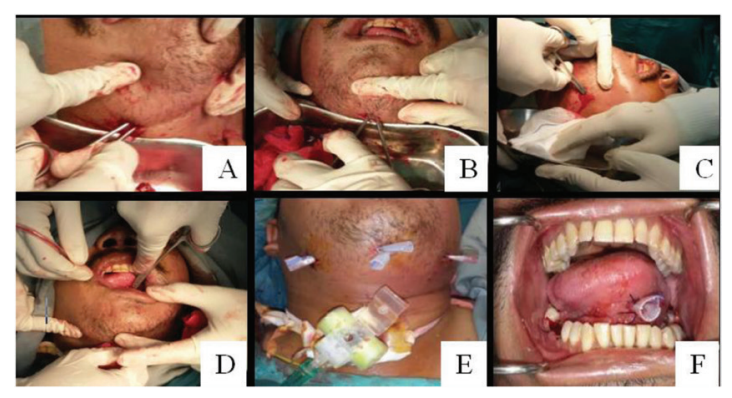

Figure 3 Intra-operative incision and drainage, A. Right submandible, B. Submentale, C. Left submandible, D. Sublingualpostoperative, E. Extraoral: rubber drain and tracheostomy cannula, F. Mouth opening turned to normal, and intraoral drain inserted


Figure 4 Clinical appearance of extra-oral and intra-oral on the 17th day. Face symmetrical; no signs of infection; mouth opening normal

\section{Discussion}

Ludwig's angina is a severe situation due to its fast development and rapid spread; it is a potentially life-threatening condition because of issues it can lead to, such as airway obstruction resulting in death. Therefore, immediate and appropriate management in the emergency unit will determine patient's condition. ${ }^{10}$ Recognizing the possibility of Ludwig's angina is the first and most important step in the diagnosis and management. There are four cardinal signs of Ludwig's angina: bilateral involvement of more than one inner tissue; gangrene with serous anguinous, slight or no pus or putrid infiltration; involvement of connective tissue, fascia, and muscle but the structure of the gland is not affected; and spreads through facial space than lymphatically. ${ }^{8}$

The diagnosis of Ludwig's angina is based primarily on conditions identified during clinical 


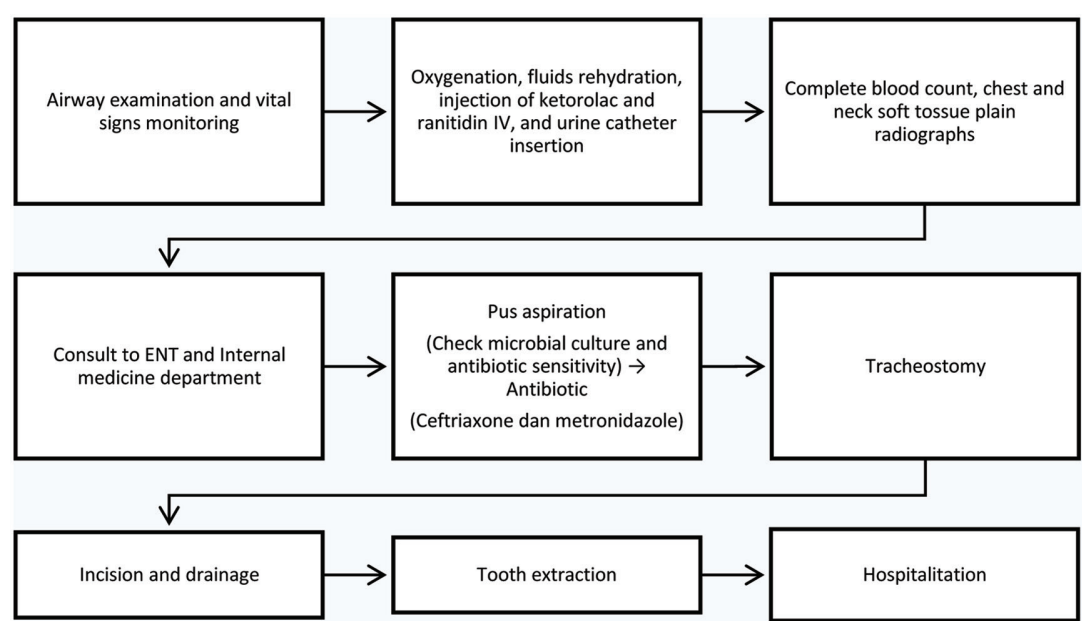

Figure 5 Algorithm of Ludwig's angina management in the ER

examination. Generally, the patient had a history of tooth extraction, poor oral hygiene, and dental pain. The symptoms included swelling and pain in the floor of the mouth and anterior of the neck, fever, dysphagia, odynophagia, drooling, trismus, dental pain and halitosis. Hoarseness, cyanosis and the position of "sniffing" (a sign of impending airway obstruction). Patients may experience dysphonia, or more specifically, the "hot potato voice" caused by edema in plica vocalist. These conditions must necessarily be spotted by the clinician because they could potentially cause severe airway obstruction. If there is diminished oxygen supply to the brain, it manifests as anxiety, agitation and confusion on the part of the patient. Intraoral examination showed visible tongue lifting, stiffness, brawny induration at the floor of mouth, and anterior neck. Lifted-off tongue can cause airway obstruction and asphyxia supraglotis if the airway is not secured. ${ }^{1,9,11}$

Sepsis is a clinical syndrome characterized by systemic inflammatory response syndrome with microbial etiology (infection). Severe sepsis occurs when the body's response to infection begins to interfere with the function of vital organs, characterized by one or more organ dysfunction, hypotension, or hypoperfusion. Septic shock occurs in cases of severe sepsis, when the blood pressure drops to a very low level and causes decreased perfusion to some vital organs. ${ }^{12}$

Management of Ludwig's angina focuses on four main actions: securing the airway, incision for drainage, antimicrobial therapy, and eliminating the focus point of infection. ${ }^{6}$ Clinician's decision should be based on careful evaluation to determine when and how to secure the airway. Surgeons must saw the signs and symptoms of respiratory disruption and interventions should be made before critical airway obstruction occurs. In this patient's case, it was decided to create an airway artificially (tracheostomy), because of impending airway obstruction, which was determined based on the symptoms this patient presented: edema of the tongue accompanied with elevation and located posteriorly, stridor, difficulty in controlling the secretion, patients' feeling more comfortable with sitting position, hot potato voice, and anxiety due to decreased oxygen supply to the brain. ${ }^{6,9,13}$

Elective tracheostomy with local anesthesia is the gold standard for definitive airway obstruction. 1 When the patient receives antibiotics, fluid resuscitation, and becomes hemodynamically stable, the source control of infection, if necessary, should be done immediately. The infected area, necrotic tissue, and tools that can potentially cause infection can all serve as a permanent source of sepsis and need to be taken care of because antimicrobials have limited penetration capabilities. ${ }^{12}$ Incision drainage aims to decompress, evacuate the pus, and prevent the spread of infection to the other internal spaces. ${ }^{5}$ Antibiotics that include comprehensive pathogens have to be administered immediately in the first hour of the initial resuscitation. There is evidence that the administration of antibiotics is adequate in the first hour of resuscitation correlated with mortality. Making appropriate culture before antibiotics is essential to confirm infection and pathogens as well as essential for consideration of de-escalation of antibiotic therapy after receiving the results of antibiotic sensitivity. ${ }^{12}$ Results of bacterial culture showed pseudomonas aeroginous, which is an opportunistic gram-negative aerobic pathogen, which is normally present in the intestine and the skin.

\section{Conclusion}

Ludwig's angina is a rare form of severe diffuse cellulitis, is acute and spreads rapidly, resulting in an emergency caused by impending airway. In advanced cases, its management includes maintaining the airway, incision drainage, antimicrobial therapy and eliminating the focus of infection. Airway management is the gold standard and primarily for Ludwig's angina. In this case, patient showed a significant improvement due to rapid and immediate management in securing the airway patency and preventing the spread of infection and further complications.

\section{Conflict of Interest}

The authors report no conflict of interest.

\section{References}

1. Barton ED, Bair AE. Ludwig's angina. Selected topics; difficult airway. J Emer Med 2008;34: 163-169. 
2. Balasubramanian S, Elavenil P, Shanmugasundaram S, et al. Ludwig's angina: a case report dan review of management. SRM J Res Dent Sciences 2014;5: 211-214.

3. Ansari NA, Khyani IAM, Ahmed S, et al. Management of Ludwig's angina: A multi centric study. Pakistan J Otolaryngol 2013;29: 3-5.

4. Nagaria A, Malliwal A, Doshi CM, et al. Ludwig's angina: a case report and review of airway management options. Indian J Basic and Applied Med Res 2015;4: 266-268.

5. Hasan W, Leonard D, Rusell J. Ludwig's angina- a controversial surgical emergency: how we do it. Case report. Int J Otolaringol 2011; 1-3.

6. Kulkarni AH, Pai-Swarupa D, Bhattarai B, et al. Ludwig's angina and airway considerations: a case report. Cases Journal 2008;1: 1-4.

7. Melo TAF, Ricker T, Carmo MPD, et al. Ludwig's angina: diagnosis and treatment. Literature Review Article. RSBO 2013;10: 172-175.

8. Lemonick DM, Ludwig's angina: diagnosis and treatment. Clinical review article. J Hosp Physician 2002; 31-37.

9. Kuriyama T, Lewis MAO, Williams DW. Oral and maxillofacial surgery. In Andersson L, Kahnberg KE, Pogrel M,
Anthony, editors. Infections of the oral and maxillofacial region. UK: Wiley-Blackwell Pub; 2010. p. 512-513.

10. Barton ED, Bair AE. Ludwig's angina. Selected topics; difficult airway. J Emer Med 2008;34: 163-169.

11. Doerr TD. Bailey's head and neck otolaryngology. 5thed. In Jhonson JT, Rosen CA, editors. Odontogenic infections. Philadelphia: Lippincott Williams and Willkins; 2014. p. 777-778.

12. Dellinger RP, Levy MM, Rhodes A, et al. Surviving sepsis campaign: international guidelines for management of severe sepsis and septic shock: 2012. J Crit Care Med 2013;41: 585-586.

13. Furst IM, Ersil P, Caminiti M. A rare complication of tooth abscess-Ludwig's angina and mediastinitis. J Can Dent Assoc 2001;67: 324-327.

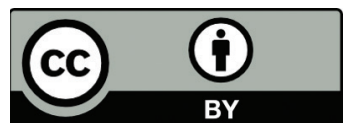

This work is licensed under a Creative Commons Attribution 